\title{
Differences in serum potassium concentrations between Chinese, Indians and Malays
}

\author{
Robert C. Hawkins* \\ Department of Laboratory Medicine, Tan Tock Seng \\ Hospital, Singapore
}

\begin{abstract}
Background: It has been suggested that potassium concentrations may vary between different geographical regions, possibly reflecting ethnic differences in potassium status. This study compared the serum potassium concentrations of three Asian ethnicities in a single geographical location.

Methods: Details of simultaneous serum potassium, creatinine, cholesterol, triglyceride and serum index measurements for samples from polyclinics and health screening were extracted for multivariable linear regression. Haemolysed and duplicate patient samples were excluded. Separate analysis was performed based on measurement platform (Roche or Beckman-Coulter) and patient location.

Results: Eighty-five thousand nine hundred and ninety-seven records met the inclusion criteria. When controlled for age, gender, serum creatinine, cholesterol and triglyceride, the average serum potassium concentration in Indians was 0.13-0.16 $\mathrm{mmol} / \mathrm{L}$ higher than in Malays, who in turn had average serum potassium concentrations $0.05-0.06 \mathrm{mmol} / \mathrm{L}$ higher than Chinese when controlled for age, gender, serum creatinine, cholesterol and triglyceride concentrations. For patients undergoing health screening, the average serum potassium concentration in Indians and Malays was 0.12 $\mathrm{mmol} / \mathrm{L}$ higher than in Chinese.

Conclusions: Chinese individuals have lower average serum potassium concentrations than Indians and Malays. This may have clinical implications in relation to the high occurrence of thyrotoxic hypokalaemic paralysis and the aetiology of sudden unexplained death syndrome (SUDS) in Asians.

Clin Chem Lab Med 2010;48:105-8.
\end{abstract}

Keywords: Asian; death; potassium; sudden; thyrotoxicosis.

\section{Introduction}

Potassium is the most abundant cation in the body, and disturbances in potassium homeostasis can be life threatening (1). It has been suggested that potassium concentrations may

*Corresponding author: Dr. Robert Hawkins, Department of Laboratory Medicine, Tan Tock Seng Hospital, 11 Jalan Tan Tock Seng, 308433 Singapore

Phone: $+(65)$ 63578943, Fax: $+(65) 62536507$,

E-mail: Robert_Hawkins@ttsh.com.sg

Received July 3, 2009; accepted September 8, 2009;

previously published online November 25, 2009 vary between different geographical regions, possibly reflecting ethnic differences in potassium status (2). The goal of this study was to compare the serum potassium concentrations of different Asian ethnic groups (Chinese, Indian and Malay) in a single geographical location.

\section{Materials and methods}

This was a retrospective study using laboratory records from a 1400 bed hospital laboratory servicing hospital inpatients, hospital outpatients and community polyclinic (primary healthcare) patients. Results from two different requesting locations were chosen as most representative of a healthy ambulatory reference population: individuals attending one of the three government polyclinics served by the laboratory and individuals from the health enrichment clinic (HEC) undergoing private "health screening" testing. Data in the laboratory information system were available from June 2005 to December 2008. Between June and October 2005, the laboratory used Roche Hitachi DP and DP clinical chemistry analysers (Roche Diagnostics, Singapore), which were replaced by Beckman-Coulter LX20 PRO and DxC 800 clinical chemistry analysers (BeckmanCoulter, Singapore) in November 2005. Therefore, separate analyses of results from the Roche platforms ("Roche dataset") and Beckman-Coulter platforms ("Beckman dataset") were performed.

The results of all serum potassium measurements with matched serum creatinine, cholesterol, triglyceride and serum index measurements, performed between June 2005 and December 2008, were extracted from the laboratory database for samples from the locations mentioned above, together with details of patient age, gender, race and location. Creatinine measurements were required to control for differences in renal function, while cholesterol and triglyceride concentrations were used to control for any potential pseudohypokalaemic effects due to increased lipid concentrations; both the Roche and Beckman-Coulter platforms use indirection selective electrode technology to measure potassium. Records were first filtered in Microsoft Excel 2000 to exclude samples with a haemolysis index $\geq 1$ (Beckman-Coulter) or $\geq 0.5 \mathrm{~g} / \mathrm{L}$ (Roche). Records were next filtered in Microsoft Access 2000 to exclude patient duplicates and ensure that only the earliest sample on each patient was included in the study. The records were divided into groups based on measurement platform (Roche or Beckman-Coulter) and patient location (polyclinic or health enrichment centre) for separate analysis. Multivariable linear regression analysis was performed on each data set for serum potassium as the outcome variable, and race, age (in years), gender (male as the reference group), serum creatinine, cholesterol and triglyceride concentrations as the predictor variables using SPSS v12 (SPSS Inc, Chicago, Illinois).

\section{Results}

There were a total of 85,997 result records which met the inclusion criteria for the study (Beckman polyclinic 64,361, Beckman HEC 6073, Roche polyclinic 15,400, Roche HEC 
163). The Roche HEC dataset was too small for analysis and was not considered further. The details of the three datasets are shown in Table 1. The Table shows lower mean potassium concentrations for Chinese individuals compared to Indian and Malays, for both locations and both analytical platforms.

The results from multivariable linear regression are shown in Table 2. For the polyclinic population, the average serum potassium concentration in Indians is $0.13-0.16 \mathrm{mmol} / \mathrm{L}$ higher than in Malays, who in turn have average serum potassium concentrations $0.05-0.06 \mathrm{mmol} / \mathrm{L}$ higher than Chinese, when controlled for age, gender, serum creatinine, cholesterol and triglyceride concentrations. The average serum potassium concentration in Indians is therefore 0.19-0.21 mmol/L higher than in Chinese. For the HEC population, the average serum potassium concentration in Indians and Malays is $0.12 \mathrm{mmol} / \mathrm{L}$ higher than in Chinese. However, there was no significant difference between Malays and Indians. Thus, all three datasets show lower serum potassium concentrations in Chinese compared to Indians and Malays, irrespective of analytical platform and patient location when controlled for age, gender, serum creatinine, cholesterol and triglyceride concentrations.

\section{Discussion}

There are few published studies on ethnic and/or geographical differences in potassium concentrations. Reidenberg et al. showed lower serum potassium concentrations in Chinese men (mean $3.82 \mathrm{mmol} / \mathrm{L}$ ) compared to Brazilians (4.06 $\mathrm{mmol} / \mathrm{L})$, Austrians (4.14 $\mathrm{mmol} / \mathrm{L})$, Dominicans (4.37 $\mathrm{mmol} / \mathrm{L}$ ), or Americans (4.38 $\mathrm{mmol} / \mathrm{L})$ (2). Similar to the present work, all analyses were performed at a single site. However, there were limited numbers in their study (Austria $\mathrm{n}=30$, China 53, Brazil 100, the Dominican Republic 38, US 103), only men 18-55 years old were included, sample haemolysis was not considered, groups were separated based on geographical location rather than ethnicity and frozen serum, transported to a separate site, was used. In contrast, Chao found no difference between serum potassium concentrations in healthy Chinese and Americans (3). A nutritional survey in China found a mean plasma potassium of $4.51 \mathrm{mmol} / \mathrm{L}$, which is higher than the values seen in the present study (2). In a single Indian study, Parab et al. showed a relatively high average potassium concentration of $4.90 \mathrm{mmol} / \mathrm{L}$, but there was no comparison data with other ethnicities (4). Arumanayagam et al. showed no difference

Table 1 Characteristics of datasets.

\begin{tabular}{lllrllllll}
\hline Location & Platform & Race & No. & $\begin{array}{l}\text { Mean age, } \\
\text { years (SD) }\end{array}$ & $\begin{array}{l}\text { Male, } \\
\%\end{array}$ & $\begin{array}{l}\text { Mean } \\
\text { potassium, } \\
\text { mmol/L (SD) }\end{array}$ & $\begin{array}{l}\text { Mean } \\
\text { creatinine, } \\
\mu m o l / L ~(S D)\end{array}$ & $\begin{array}{l}\text { Mean } \\
\text { cholesterol, } \\
\text { mmol/L (SD) }\end{array}$ & $\begin{array}{l}\text { Mean } \\
\text { triglyceride, } \\
\text { mmol/L (SD) }\end{array}$ \\
\hline Polyclinic & Roche & Chinese & 13,465 & $64.0(11.2)$ & 45 & $4.37(0.46)$ & $83.6(30.5)$ & $5.12(0.92)$ & $1.65(1.01)$ \\
& & Malay & 802 & $59.5(11.2)$ & 43 & $4.42(0.50)$ & $83.9(31.5)$ & $5.20(1.04)$ & $1.77(1.02)$ \\
& & Indian & 1133 & $60.8(12.2)$ & 45 & $4.57(0.43)$ & $80.2(32.6)$ & $4.98(0.94)$ & $1.63(1.03)$ \\
Polyclinic & \multirow{2}{*}{ Beckman } & Chinese & 55,871 & $64.2(11.3)$ & 46 & $4.29(0.47)$ & $84.9(33.7)$ & $5.29(0.96)$ & $1.43(0.90)$ \\
& & Malay & 3444 & $60.2(12.0)$ & 41 & $4.37(0.50)$ & $87.9(44.4)$ & $5.48(1.13)$ & $1.56(1.01)$ \\
HEC & \multirow{2}{*}{ Beckman } & Indian & 5046 & $60.7(12.0)$ & 45 & $4.48(0.46)$ & $81.0(31.8)$ & $5.10(1.01)$ & $1.48(1.24)$ \\
& & Chinese & 5656 & $45.6(12.7)$ & 48 & $4.16(0.44)$ & $74.6(18.7)$ & $5.47(0.96)$ & $1.19(0.78)$ \\
& & Malay & 168 & $41.1(12.1)$ & 63 & $4.29(0.47)$ & $81.3(17.3)$ & $5.74(1.21)$ & $1.58(1.28)$ \\
& & Indian & 249 & $43.1(11.6)$ & 65 & $4.28(0.45)$ & $80.6(15.9)$ & $5.25(0.95)$ & $1.43(0.97)$ \\
\hline
\end{tabular}

SD, standard deviation; HEC, Health Enrichment Centre.

Table 2 Results of multivariable linear regression controlled for age, gender, serum creatinine, cholesterol and triglyceride concentrations.

\begin{tabular}{lllr}
\hline Predictor variable & $\begin{array}{l}\text { Adjusted difference in mean } \\
\text { potassium, mmol/L }\end{array}$ & $\begin{array}{l}\text { Standard error } \\
(\mathrm{K} \text { in mmol/L) }\end{array}$ \\
\hline Roche polyclinic & & & \\
$\quad$ Indian (vs. Chinese) & 0.206 & 0.014 & $<0.001$ \\
$\quad$ Indian (vs. Malay) & 0.160 & 0.013 & $<0.001$ \\
$\quad$ Malay (vs. Chinese) & 0.046 & 0.017 & $<0.001$ \\
Beckman polyclinic & & & \\
$\quad$ Indian (vs. Chinese) & 0.191 & 0.007 & $<0.001$ \\
$\quad$ Indian (vs. Malay) & 0.127 & 0.008 & $<0.001$ \\
$\quad$ Malay (vs. Chinese) & 0.064 & 0.008 & $<0.001$ \\
Beckman HEC & & & $<0.001$ \\
$\quad$ Indian (vs. Chinese) & 0.122 & 0.029 & 0.999 \\
Indian (vs. Malay) & $<0.001$ & 0.044 & $<0.001$ \\
Malay (vs. Chinese) & 0.122 & 0.035 & \\
\hline
\end{tabular}

HEC, Health Enrichment Centre. 
in erythrocyte sodium content, sodium pump activity (including sodium-potassium ATPase activity), sodium-lithium countertransport and sodium-potassium co-transport activities between European males, non-Chinese Asian males and Chinese males (5). However, sodium-lithium countertransport was higher and sodium-potassium co-transport was lower in Chinese compared to either the Europeans or non-Chinese Asians.

The present study provides clear evidence of differences in serum potassium concentration between Asian ethnic groups. Chinese individuals had the lowest mean potassium concentrations of the three races studied across different analytical platforms and patient populations. In the polyclinic population, the highest mean potassium concentrations were seen in Indians, followed by Malay and Chinese individuals. In the HEC group, there was no difference between potassium concentrations in Indians and Malays, but Chinese again showed the lowest concentrations. Sample collection, handling, transport and analysis were identical for all samples, ruling out pre-analytical or analytical causes as a source of these differences. Potassium concentrations have been shown to be lower during the monsoon season in India than at other times, but the single geographical location of all subjects in this study ruled out climate variation as a factor (2). The study considered potential confounding factors such as, age, gender, renal function and hyperlipidaema, but information on additional patient characteristics such as medication, body mass index and clinical conditions were not available and limited to that in the laboratory database. It is possible that such factors may explain the differences seen, but the presence of lower potassium concentrations in Chinese in both the polyclinic (chronically ill) and HEC (apparently healthy) population suggests this is a true finding.

Although the pattern of lower potassium concentrations in Chinese was seen in both patient populations studied, the magnitude differed $(0.20 \mathrm{mmol} / \mathrm{L}$ lower in polyclinic patients vs. $0.12 \mathrm{mmol} / \mathrm{L}$ in HEC patients) and the difference between Malays and Indians in the polyclinic group was not present in the HEC population. Mean serum potassium concentrations in the HEC group were also uniformly lower than those seen in the polyclinic groups. This latter probably reflects unequal delay times between sample collection and centrifugation, with HEC samples being collected on-site at the hospital, while polyclinic samples are collected off-site and $<30 \%$ arriving at the laboratory within $2 \mathrm{~h}$ of collection. Inspection of Table 1 shows that HEC patients were younger than those in the polyclinic group, with a greater proportion of Indian and Malay men. Significant socio-economic differences would also be expected between individuals in the two groups. Polyclinics provide cheap but limited primary care based in the community for the bulk of the Singaporean population, while HEC offers relatively expensive comprehensive hospital-based health screening packages designed for the more affluent. Although age and gender differences between the two groups were accounted for in the regression model, lifestyle and dietary factors may account for the differences observed. A possible explanation is that the more affluent HEC patients eat more relatively expensive Western or non-traditional foods than the polyclinic patients who follow traditional Asian diets. Another explanation may be that HEC patients are more health conscious, as evidenced by their decision to undergo health screening, and are choosing "healthier', or different dietary items within the traditional Asian dietary menu compared to polyclinic patients. Detailed dietary and lifestyle studies would be needed to investigate this further and clarify the importance of lifestyle vs. physiological factors to explain the differences seen.

Physiological and/or dietary differences may explain the racial differences seen here. Ethnic differences in plasma folate, vitamin $B_{12}$, vitamin $C$ and selenium have previously been shown to vary between the racial groups in Singapore, probably based on food and cooking practices $(6,7)$. Liquorice contains glycyrrhizinic acid that can cause hypokalaemia and is used in Asia for culinary and traditional medical purposes. Differences in liquorice consumption between ethnic groups may help explain the variation in serum potassium concentration.

Despite the small magnitude of the differences in potassium concentration, there may be clinical implications in relation to hypokalaemic periodic paralysis and sudden unexplained death syndrome (SUDS) in Asians. Hypokalaemic periodic paralysis is a disorder of unknown origin characterised by potentially fatal episodes of respiratory muscle paralysis (8). Sudden movement of potassium into cells, precipitated by insulin or catecholeamine release (stress, rest after exercise, carbohydrate intake) can lower the serum potassium concentration to $1.5-2.5 \mathrm{mmol} / \mathrm{L}$. Asian males are particularly at risk for hypokalaemic periodic paralysis, with an estimated risk of $15 \%-20 \%$ in Chinese individuals with hyperthyroidism (8).

SUDS is the death of apparently healthy individuals, usually young men, in whom postmortem examination does not reveal the cause of death. The victims are in apparently good health and usually die at night while sleeping. They die within minutes after the onset of agonal respiration. Patients who have been resuscitated were found to have ventricular fibrillation and inducible polymorphic ventricular tachycardia in the electrophysiologic laboratory. This syndrome has been described most frequently in young Southeast Asian men (9). Such deaths during sleep are a leading cause of death of young men in rural northeastern Thailand (known as "laitai", in the local dialect), and the potential role of hypokalaemia has been well studied (10). Tosukhowong et al. documented low serum potassium concentrations in construction workers from north-east Thailand, conjecturing that this might be a trigger factor for ventricular fibrillation and explain the increased risk of SUDS in this population (11). His group have documented low erythrocyte sodium-potassium ATPase in relatives of patients dying from SUDS and suggested that the pathogenesis of SUDS could be related to hypokalaemia and possibly an acquired membrane sodium-potassium pump defect $(12,13)$. Low potassium intake may also relate to the low serum concentrations seen in this population (14).

In summary, this study shows consistently lower average serum potassium concentrations in Chinese individuals com- 
pared to Indians and Malays. These lower concentrations may be a predisposing factor to hypokalaemic periodic paralysis and sudden unexplained death seen in Asians. This study also illustrates the need to distinguish between different Asian ethnicities when performing health research in Asian populations.

\section{Conflict of interest statement}

Authors' conflict of interest disclosure: The authors stated that there are no conflicts of interest regarding the publication of this article.

Research funding: None declared.

Employment or leadership: None declared.

Honorarium: None declared.

\section{References}

1. Halperin ML, Kamel KS. Potassium. Lancet 1998;352:135-40.

2. Reidenberg MM, Gu ZP, Lorenzo B, Coutinho E, Athayde C, Frick J, et al. Differences in serum potassium concentrations in normal men in different geographic locations. Clin Chem 1993; 39:72-5.

3. Chao TJ. Comparison of serum sodium and potassium by direct ion-selective electrode analysis between Chinese and American people. Proc Natl Sci Counc Repub China B 1987;11:97-9.

4. Parab PB, Kulkarni BS, Satoskar RS. Total body exchangeable potassium in apparently healthy men from warm and humid climate. Indian J Med Sci 1973;27:843-8.

5. Arumanayagam M, MacDonald D, Swaminathan R. Differences in erythrocyte cation (sodium) transport between Chinese and non Chinese males. Clin Exp Hypertens A 1987;9:719-39.

6. Hughes K, Ong CN. Vitamins, selenium, iron, and coronary heart disease risk in Indians, Malays, and Chinese in Singapore. J Epidemiol Community Health 1998;52:181-5.

7. Hughes K, Ong CN. Homocysteine, folate, vitamin B12, and cardiovascular risk in Indians, Malays, and Chinese in Singapore. J Epidemiol Community Health 2000;54:31-4.

8. Fontaine B, Lapie P, Plassart E, Tabti N, Nicole S, Reboul J, et al. Periodic paralysis and voltage-gated ion channels. Kidney Int 1996;49:9-18.

9. Veerakul G, Nademanee K. What is the sudden death syndrome in Southeast Asian males? Cardiol Rev 2000;8:90-5.

10. Tatsanavivat $\mathrm{P}$, Chiravatkul A, Klungboonkrong V, Chaisiri S, Jarerntanyaruk L, Munger RG, et al. Sudden and unexplained deaths in sleep (Laitai) of young men in rural northeastern Thailand. Int J Epidemiol 1992;21:904-10.

11. Tosukhowong P, Sriboonlue P, Tungsanga K, Bovornpadungkitti S, Chatuporn S, Muktahant B, et al. Potassium status of Northeast Thai constructors in three different geographic locations. J Med Assoc Thai 2001;84(Suppl 1):S163-72.

12. Tosukhowong $\mathrm{P}$, Chotigasatit $\mathrm{C}$, Tungsanga K, Sriboonlue $\mathrm{P}$, Pansin P, Sitprija V. Hypokalemia, high erythrocyte $\mathrm{Na}+$ and low erythrocyte $\mathrm{Na}$, K-ATPase in relatives of patients dying from sudden unexplained death syndrome in north-east Thailand and in survivors from near-fatal attacks. Am J Nephrol 1996;16:369-74.

13. Tosukhowong P, Tungsanga K, Kittinantavorakoon C, Chaitachawong C, Pansin P, Sriboonlue P, et al. Low erythrocyte Na/ K-pump activity and number in northeast Thailand adults: evidence suggesting an acquired disorder. Metabolism 1996;45: 804-9.

14. Sriboonlue P, Prasongwatana V, Suwantrai S, Bovornpadungkitti S, Tungsanga K, Tosukhowong P. Potassium contents of northeastern Thai foods. J Med Assoc Thai 1998;81:616-26. 